\title{
A novel approximate dynamic programming approach for constrained equipment replacement problems: A case study
}

\author{
Sadeghpour, H. ${ }^{a}$, Tavakoli, A. ${ }^{\text {a, }}$, Kazemi, M. ${ }^{a}$, Pooya, A. ${ }^{a}$ \\ ${ }^{a}$ Ferdowsi University of Mashhad, Faculty of Economics and Administrative Science, Mashhad, Iran
}

\begin{abstract}
A B S T R A C T
This paper presents a novel Approximate Dynamic Programming (ADP) approach to solve large-scale nonlinear constrained Equipment Replacement (ER) problems. Since ADP requires accurate estimations of states for future periods, a heuristic estimator based on data clustering was developed for the case study of this paper with small number of sampling periods. This ADP approach uses a Rollout Algorithm to formulate the problem in a Rolling horizon. The model was solved using Genetic Algorithm (GA). This framework was successfully applied for the decision making process of replacement/maintenance of 497 transformers in a power distribution company, which led to a significant reduction in the expected costs. The proposed framework possesses favourable features such as minimizing the effect of uncertainties in the state variables and measurement inaccuracies, which make the model robust and reliable. This work provides a novel general approach that can be employed for other industrial cases and operations research problems.
\end{abstract}

(c) 2019 CPE, University of Maribor. All rights reserved.

\begin{tabular}{l} 
A R T I C L E I N F O \\
\hline Keywords: \\
Equipment replacement; \\
Approximate dynamic program- \\
ming; \\
Rollout algorithm; \\
State estimation; \\
Genetic algorithm \\
*Corresponding author: \\
tavakoli-a@ um.ac.ir \\
(Tavakoli, A.) \\
Article history: \\
Received 7 May 2019 \\
Revised 8 September 2019 \\
Accepted 10 September 2019
\end{tabular}

\section{References}

[1] Fan, W., Haile, E., Chavez, T., Radley, L., Sorbet, R., Eifert, A., Machemehl, R., Gemar, M., Murshed, N., Yu, Y. (2013). Equipment replacement/retention decision making: Final report, College of Engineering and Computer Science, The University of Texas at Tyler, Texas, USA, from https://library.ctr.utexas.edu/ctr-publications/0-6693-1.pdf, accesed May 7, 2019.

[2] Alvarado, M.A., Rocha, S.L. (2018). Development of methodology of evaluation for medical equipment replacement for developing countries, In: Lhotska, L., Sukupova, L., Lacković, I., Ibbott, G. (eds.), World Congress on Medical Physics and Biomedical Engineering 2018, IFMBE Proceedings, Vol. 68/3, Springer, Singapore, doi: $10.1007 /$ 978-981-10-9023-3 69 .

[3] Zvipore, D.C., Nyamugure, P., Maposa, D., Lesaoana, M. (2015). Application of the equipment replacement dynamic programming model in conveyor belt replacement: Case study of a gold mining company, Mediterranean Journal of Social Sciences, Vol. 6, No. 2, 605-612, doi: 10.5901/miss.2015.v6n2s1p605.

[4] Hara, M., Tanaka, J., Okazaki, H., Odani, K., Tamura, K., Teramae, T. (2016). Completion of "Joyo" experimental fast reactor upper core structure replacement-development of fast reactor core internal equipment replacement method, Mitsubishi Heavy Industries Technical Review, Vol. 53, No. 4, 65-74.

[5] Ukwu, C., Dazel, F.I., Ozemelah, I.J. (2017). Optimal equipment replacement strategies with index one electronic implementations: A case study of Zenith processor tin mining company, Jos, Plateau State, Nigeria, GEInternational Journal of Engineering Research, Vol. 5, No. 7, 14-28.

[6] Karro, D., Mahler, R. (2018). Information technology equipment replacement calculation systems and methods, US patent, Patent No. US20180253709.

[7] Leung, L.C., Tanchoco, J.M.A. (1990). Multiple machine replacement analysis, Engineering Costs and Production Economics, Vol. 20, No 3, 265-275, doi: 10.1016/0167-188X(90) 90074-R.

[8] Sethi, S., Sorger, G. (1991). A theory of rolling horizon decision making, Annals of Operations Research, Vol. 29, No. 1, 387-415, doi: 10.1007/BF02283607. 
[9] Fraser, J.M., Posey, J.W. (1989). A framework for replacement analysis, European Journal of Operational Research, Vol. 40, No. 1, 43-57, doi: 10.1016/0377-2217(89)90271-3.

[10] Boucekkine, R., Germain, M., Licandro, O. (1997). Replacement echoes in the vintage capital growth model, Journal of Economic Theory, Vol. 74, No. 2, 333-348, doi: 10.1006/jeth.1996.2265.

[11] Hritonenko, N., Yatsenko, Y. (2009). Integral equation of optimal replacement: Analysis and algorithms, Applied Mathematical Modelling, Vol. 33, No. 6, 2737-2747, doi: 10.1016/i.apm.2008.08.007.

[12] Motamedi, N., Hadizadeh, M., Peyghami, M.R. (2014). A nonlinear integral model of optimal replacement: Numerical viewpoint, Communications in Numerical Analysis, Vol. 2014, Article ID: cna-00207, 9 pages, doi: 10.5899/ 2014/cna-00207.

[13] Jakobsen, I.M. (2018). Cost predictive model to support decision making regarding equipment replacement, using system analysis and system dynamics: A case study in Hydro Aluminium Karmøy, Master's thesis, University of Stavanger, Norway.

[14] Fan, W.D., Gemar, M.D., Machemehl, R. (2013). Equipment replacement decision making: Opportunities and challenges, Journal of the Transportation Research Forum, Vol. 52, No. 3, 79-90.

[15] Bellman, R.E. (1953). An introduction to the theory of dynamic programming, RAND Corporation, Santa Monica, California, USA.

[16] Bellman, R. (1955). Equipment replacement policy, Journal of the Society for Industrial and Applied Mathematics, Vol. 3, No. 3, 133-136, doi: 10.1137/0103011.

[17] Hillier, F.S., Lieberman, G.J. (2015). Introduction to operations research, 10th edition, McGraw-Hill Education, New York, USA.

[18] Bertsekas, D.P. (2005). Dynamic programming and optimal control, 3rd edition, Athena Scientific, Massachusetts, USA.

[19] Karns, R., Zhang, G., Jeran, N., Havas-Augustin, D., Missoni, S., Niu, W., Indugula, S.R., Sun, G., Durakovic, Z., Smolej Narancic, N., Rudan, P., Chakraborty, R., Deka, R. (2011). Replication of genetic variants from genome-wide association studies with metabolic traits in an island population of the Adriatic coast of Croatia, European Journal of Human Genetics, Vol. 19, No. 3, 341-346, doi: 10.1038/ejhg.2010.178.

[20] Box, G.E.P., Meyer, R.D. (1986). Dispersion effects from fractional designs, Technometrics, Vol. 28, No. 1, 19-27, doi: 10.1080/00401706.198.10488094.

[21] Milligan, G.W., Cooper, M.C. (1988). A study of standardization of variables in cluster analysis, Journal of classification, Vol. 5, No. 2, 181-204, doi: 10.1007/BF01897163.

[22] Naderian, A., Cress, S., Piercy, R., Wang, F., Service, J. (2008). An approach to determine the health index of power transformers, In: Conference Record of the 2008 IEEE International Symposium on Electrical Insulation, Vancouver, Canada, 192-196, doi: 10.1109/ELINSL.2008.4570308.

[23] Ishikawa, A., Amagasa, M., Shiga, T., Tomizawa, G., Tatsuta, R., Mieno, H. (1993). The max-min Delphi method and fuzzy Delphi method via fuzzy integration, Fuzzy Sets and Systems, Vol. 55, No. 3, 241-253, doi: 10.1016/01650114(93)90251-C.

[24] Lee, A.H.I., Wang, W.-M., Lin, T.-Y. (2010). An evaluation framework for technology transfer of new equipment in high technology industry, Technological Forecasting and Social Change, Vol. 77, No 1, 135-150, doi: 10.1016/ ..techfore.2009.06.002.

[25] Fan, W.D., Machemehl, R., Gemar, M., Brown, L. (2014). A stochastic dynamic programming approach for the equipment replacement optimization under uncertainty, Journal of Transportation Systems Engineering and Information Technology, Vol. 14, No. 3, 76-84, doi: 10.1016/S1570-6672(13)60137-3. 


\section{APEM}

\title{
Nov pristop približnega dinamičnega programiranja za problem zamenjave opreme z omejitvami: Študija primera
}

\author{
Sadeghpour, H. ${ }^{\text {a }}$, Tavakoli, A. ${ }^{a}{ }^{,}$, Kazemi, M. ${ }^{a}$, Pooya, A. ${ }^{a}$ \\ ${ }^{a}$ Ferdowsi University of Mashhad, Faculty of Economics and Administrative Science, Mashhad, Iran
}

\section{POVZETEK}

Ta članek predstavlja nov pristop približnega dinamičnega programiranja (ADP) za reševanje velikih nelinearnih problemov z zamenjavo opreme (ER). Ker ADP zahteva natančne ocene stanja za prihodnja obdobja, je bil v tem prispevku za študijo primera razvit hevristični ocenjevalec z majhnim številom obdobij vzorčenja, ki temelji na združevanju podatkov. Za oblikovanje problema s premakljivim obzorjem, uporablja ta pristop ADP algoritem rollout. Model smo rešili z uporabo genetskega algoritma (GA). Ta raziskovalni okvir je bilo uspešno uporabljen za postopek odločanja o zamenjavi/vzdrževanju 497 transformatorjev v elektrodistribucijskem podjetju, kar je povzročilo znatno zmanjšanje pričakovanih stroškov. Predlagani okvir ima ugodne lastnosti, kot sta zmanjšanje učinka negotovosti v spremenljivkah stanja in netočnosti meritev, zaradi katerih je model robusten in zanesljiv. Raziskava ponuja nov splošen pristop, ki ga je mogoče uporabiti za druge industrijske primere in reševanje problemov pri operacijskih raziskavah.

(c) 2019 CPE, University of Maribor. All rights reserved.

\section{PODATKI O ČLANKU}

Ključne besede:

Zamenjava opreme;

Približno dinamično programiran-

je;

Algoritem rollout;

Ocena stanja;

Genetski algoritem

* Kontaktna oseba:

tavakoli-a@um.ac.ir

(Tavakoli, A.)

Zgodovina članka:

Prejet 7. maja 2019

Popravljen 8. septembra 2019

Sprejet 10. septembra 2019 